\title{
SINGULAR REGULAR NEIGHBORHOODS AND LOCAL FLATNESS IN CODIMENSION ONE
}

\author{
ROBERT J. DAVERMAN ${ }^{1}$
}

\begin{abstract}
For an $(n-1)$-manifold $S$ topologically embedded as a closed subset of an $n$-manifold $N$, we define what it means for $S$ to have a singular regular neighborhood in $N$. The principal result demonstrates that $S$ has a singular regular neighborhood in $N$ if and only if the homotopy theoretic condition holds that $N-S$ is locally simply connected (1-LC) at each point of $S$. Consequently, $S$ has a singular regular neighborhood in $N$ if and only if $S$ is locally flatly embedded $(n \neq 4)$.
\end{abstract}

In 1964 Hempel [7] obtained such results for the case $n=3$; for a 2manifold $\Sigma$ in the 3-sphere $S^{3}$, the term he used, namely, that $\Sigma$ could be deformed into each of its complementary domains, is equivalent to the property that $\Sigma$ has a singular regular neighborhood in $S^{3}$. His argument for showing that $S^{3}-\Sigma$ is 1 -LC at each point of $\Sigma$ was based on the Sphere Theorem, a result that applies only in dimension 3, and, therefore, his proof does not extend to higher dimensions. The argument given here, which provides an alternative to Hempel's for $n=3$, employs the important result of Olum [12] that a degree one map between two orientable $n$-manifolds induces an epimorphism of their fundamental groups.

The notion of singular regular neighborhood has arisen in another setting: Cannon [2, Theorem 3.10] has shown that a 1-complex in a 3-manifold is tame if and only if it has a singular regular neighborhood. However, we consider no analogues of his result and deal only with singular regular neighborhoods of codimension one submanifolds. (Bryant and Lacher [16] have described an extension of this property, which they call strong freeness, that applies to submanifolds of arbitrary codimension.)

1. Definitions and notation. All manifolds are connected and boundaryless. If we refer to an $(n-1)$-manifold $S$ in an $n$-manifold $N$, we assume $S$ to be embedded as a closed subset of $N$.

Cohomology groups are computed with integer coefficients. Essentially we follow Epstein's formulation [6] of the definition of degree of a map (see also

Presented to the Society, January 17, 1974 under the title Conditions implying a codimension one manifold is locally flat; received by the editors June 21, 1974 and, in revised form, January 21, 1975.

AMS (MOS) subject classifications (1970). Primary 57A45; Secondary 57A15, 57A35, 57A40, 57A50.

Key words and phrases. Singular regular neighborhood, locally singularly collared, 1-ULC embedding, locally flat embedding, degree one mapping of manifolds.

${ }^{1}$ Research supported in part by NSF Grant GP-33872.

Copyright $\odot$ 1976, American Mathematical Society 
[12]); in particular we disregard negative values of degree and say that a proper map (i.e., the inverse image of each compact set is compact) $f: N \rightarrow M$ between orientable $n$-manifolds has degree one if $f$ induces isomorphisms between the cohomology groups with compact supports $H_{c}^{n}(M)$ and $H_{c}^{n}(N)$.

Let $S$ be an $(n-1)$-manifold in an $n$-manifold $N$ such that $N-S$ has two components $U$ and $V$. We say that $S$ has a singular regular neighborhood in $U$ if there exists a map $h$ of $S \times I$ into $\mathrm{Cl} U$ such that $h(s, 0)=s$ and $h(s, t) \in U$ for all $s \in S, t>0$, and that $S$ has a singular regular neighborhood in $N$ if it has singular regular neighborhoods in both $U$ and $V$. Similarly, we say that $S$ is locally singularly collared from $U$ at $s \in S$ if there exist an open subset $W$ of $S$ containing $s$ and a map $f$ of $W \times I$ into $\mathrm{Cl} U$ such that $f(w, 0)=w$ and $f(w, t) \in U$ for all $w \in W$ and $t>0$, and that $S$ is locally singularly bicollared at $s \in S$ if it is locally singularly collared from both $U$ and $V$ at $s$. According to standard practice, $S$ is said to be locally singularly collared from $U$ or locally singularly bicollared if it has the appropriate property at each of its points.

In the case that $S$ does not separate $N$, then $S$ has a singular regular neighborhood if there exist a locally flat embedding $g$ of $S$ in an $n$-manifold $M$, a neighborhood $N^{\prime}$ of $g(S)$ in $M$, and a map $f$ of $N^{\prime}$ into $N$ such that $f g=$ identity, $f\left(N^{\prime}-g(S)\right) \cap S=\varnothing$, and to each $s \in S$ there correspond connected neighborhoods $A_{s}$ and $B_{s}$ of $g(s)$ in $N^{\prime}$ and $s$ in $N$, respectively, such that $A_{s}-g(S)$ consists of two components $A_{1}$ and $A_{2}$ for which $f\left(A_{1}\right)$ and $f\left(A_{2}\right)$ are contained in different components of $B_{s}-S$. Note that in this case there is a natural and sensible definition of locally singularly bicollared and that if $S$ has a singular regular neighborhood, then it is locally singularly bicollared in $N$.

2. Degree one maps. Throughout this section we shall suppose that $\Sigma$ is an $(n-1)$-manifold in $E^{n}$ and that $E^{n}-\Sigma$ consists of the two components $U$ and $V$.

Lemma 1. Suppose $D$ is an $(n-1)$-cell in $\Sigma$ and $f: D \times I \rightarrow \mathrm{Cl} U$ is a map such that $f(d, 0)=d$ and $f(d, t) \in U$ for all $d \in D, t>0$. Then there exists an open subset $W$ of $\mathrm{Cl} U$ such that Int $D \subset W \subset f(D \times I)$.

Proof. Let $\Sigma^{\prime}$ denote the closure of $\Sigma$ in $S^{n}=E^{n} \cup\{\infty\}$. There exists a map $H$ of $\Sigma^{\prime} \times I$ into $\Sigma^{\prime} \cup f(D \times I)$ such that

$$
\begin{aligned}
H(s, 0) & =s & & \text { for all } s \in \Sigma^{\prime}, \\
H\left(s^{\prime}, t\right) & =s^{\prime} & & \text { for all } s^{\prime} \in \Sigma^{\prime}-\text { Int } D, \\
H\left(s^{\prime \prime}, t\right) \cap \Sigma^{\prime} & =\varnothing & & \text { for all } s^{\prime \prime} \in \operatorname{Int} D, \quad t>0 .
\end{aligned}
$$

Define $W$ as the intersection of $\mathrm{Cl} U$ and the component of $S^{n}-H\left(\Sigma^{\prime}, 1\right)$ that contains Int $D$. We show that $W \subset f(D \times I)$.

Suppose to the contrary that there exists

$$
w \in W-f(D \times I) \subset W-H\left(\Sigma^{\prime} \times I\right) .
$$

Choose a point $z \in S^{n}-\mathrm{Cl} U$ and regard $S^{n}-\{z\}$ as $E^{n}$. As in [9, p. 97], for $x \neq z$ let $\pi_{x}$ denote the radial map of $S^{n}-\{x, z\}$ onto the unit $(n-1)$-sphere 
centered at $x$. Then $\pi_{w} H_{0}$ and $\pi_{w} H_{1}$ are homotopic maps of $\Sigma^{\prime}$. However, for $y \in S^{n}-\mathrm{Cl} U, y \neq z$, we see that $w$ and $y$ belong to different components of $S^{n}-H\left(\Sigma^{\prime}, 0\right)$ and belong to the same component of $S^{n}=H\left(\Sigma^{\prime}, 1\right)$. This leads to the desired contradiction, because according to Theorem VI.10 of [9] (and its proof), $\pi_{w} H_{0}$ is an essential map and $\pi_{w} H_{1}$ is an inessential map.

Lemma 2. Suppose $q \in \Sigma$ such that $\Sigma$ is locally singularly collared from $U$ at q. Then $U$ is 1-LC at $q$.

Proof. Let $\varepsilon>0$. The hypothesis implies that there exist (i) an $(n-1)$-cell $D$ on $\Sigma$ for which $q \in$ Int $D$ and (ii) a map $f$ of $D \times[0,1]$ into $N_{\varepsilon}(q)$ such that $f(d, 0)=d$ and $f(D \times(0,1]) \subset U$. Applying Lemma 1, we determine an open subset $W$ of $\mathrm{Cl} U$ such that

$$
\text { Int } D \subset W \subset f(D \times I) \subset N_{\varepsilon}(q) \text {. }
$$

For the component $Y$ of $f^{-1}(W)$ containing Int $D \times\{0\}$, it follows immediately that $f \mid Y$ is a proper map, which implies that the degree of $f \mid Y$ is defined [6].

Defining $W_{U}=W \cap U, Y_{U}=Y \cap U$, and $f_{U}=f \mid Y_{U}$, we shall prove that $f_{U}$ is a degree one map of $Y_{U}$ to $W_{U}$. From the exact sequence

$$
\cdots \rightarrow H_{c}^{q-1}(X-A) \rightarrow H_{c}^{q}(A) \rightarrow H_{c}^{q}(X) \rightarrow H_{c}^{q}(X-A) \rightarrow \cdots
$$

of the pair $(X, A)$, where $X$ is locally compact and $A$ is open in $X$ (see [5, Chapter $\mathrm{X}]$ ), we obtain the following commutative diagram:

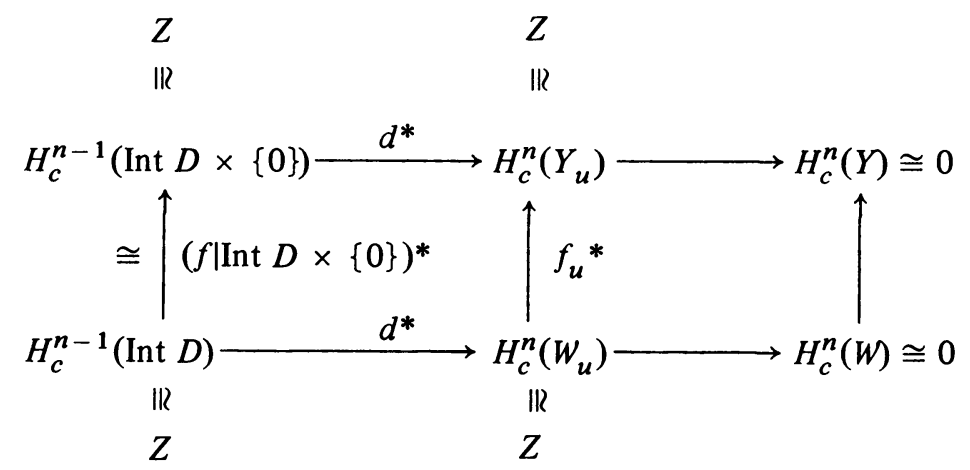

The fact that $H_{c}^{n}(Y) \cong H_{c}^{n}(W) \cong 0$ follows from [14, Theorem 6.6.10] and that the other groups are isomorphic to $Z$ holds because the spaces are orientable manifolds. This implies that the $d^{*}$ 's are isomorphisms and, therefore, that $f_{U}$ is a degree one map. Consequently, $f_{U}$ induces an epimorphism of $\pi_{1}\left(Y_{U}\right)$ to $\pi_{1}\left(W_{U}\right)$ [6, Corollary 3.4].

To each loop $\alpha$ in $W_{U}$ there corresponds a loop $\alpha^{\prime}$ in $Y_{U}$ for which $\left(f_{U}\right)\left[\alpha^{\prime}\right]=[\alpha]$. Since $\alpha^{\prime}$ is contractible in Int $D \times(0,1), \alpha$ is contractible in $f($ Int $D \times(0,1)) \subset N_{\varepsilon}(q) \cap U$. Hence, $U$ is $1-\mathrm{LC}$ at $q$.

Remark. If $\Sigma$ is locally collared from $V$ at $q$, then we can detect geometrically that the degree of $f_{U}$ is one by extending $f$ to a map $f$ of $D \times[-1,1]$ into $N_{\varepsilon}(q)$ such that $f$ sends $D \times[-1,0]$ homeomorphically onto a collar on $D$ from $V$. Because $f$ is $1-1$ on $f^{-1} f(\operatorname{Int} D \times(-1,0))$, the degree of 


$$
f \mid Y \cup(\text { Int } D \times(-1,0]): Y \cup(\text { Int } D \times(-1,0]) \rightarrow W \cup f(\operatorname{Int} D \times(-1,0])
$$

is one [6, p. 372], and it easily follows that the degree of the restriction $f_{U}$ is one also.

3. Main results. The following two results are immediate consequences of Lemma 2.

THEOREM 3. Suppose $S$ is an $(n-1)$-manifold in an $n$-manifold $N$ such that $N-S$ has two components, $U$ and $V$, and $S$ is locally singularly collared from $U$ at $s \in S$. Then $U$ is 1-LC at $s$. Thus, if $S$ has a singular regular neighborhood in $U$ (or if $S$ is locally singularly collared from $U$ ), $U$ is 1-LC at each point of $S$.

THEOREM 4. Suppose $S$ in an $(n-1)$-manifold in an $n$-manifold $N$ and $S$ is locally singularly bicollared at $s \in S$. Then $N-S$ is 1-LC at $s$. Thus, if $S$ has a singular regular neighborhood in $N$ (or if $S$ is locally singularly bicollared), then $N-S$ is 1-LC at each point of $S$.

Corollary 5. Suppose $S$ is an $(n-1)$-manifold in an $n$-manifold $N(n \neq 4)$ such that $S$ has a singular regular neighborhood in $N$ (or is locally singularly bicollared). Then $S$ is locally flat.

The corollary follows from [1, Theorem 7] in case $n=3$ and from [3] or [4] in case $n \geqq 5$.

REMARK. If $S$ separates $N$ into two components $U$ and $V$, and $U$ is 1 -LC at each point of $S$, it is not known $(n \geqslant 4)$ whether $S$ is collared from $U .^{2}$ A necessary and sufficient condition $(n \geqslant 5)$ that $S$ be collared from $U$ is that for each $\varepsilon>0$ there exist an $\varepsilon$-homeomorphism of $S$ onto a locally flat manifold in $N$ [13, Theorem 5].

Bryant and Lacher [16] have obtained analogues of Theorem 4 and Corollary 5 for $S$ a manifold of arbitrary dimension embedded in $N$.

Corollary 6. Suppose $S$ is a locally flat $(n-1)$-manifold in an $n$-manifold $N$ $(n \geqslant 4)$ and $f$ is a map of $N$ onto an $n$-manifold $N^{\prime}$ such that $f \mid S$ is 1-1 and $f(N-S)=N^{\prime}-f(S)$. Furthermore, if $f(S)$ does not separate $N^{\prime}$, suppose that $f$ is a proper map. Then $f(S)$ is locally flat.

Proof. Clearly $S$ is locally singularly bicollared in $N$. A straightforward argument shows that $f(S)$ has the same property in $N^{\prime}$.

Note that in case $f(S)$ separates $N^{\prime}$, instead of requiring that $f(N-S)$ $=N^{\prime}-f(S)$ one can require simply that $f(N-S)$ be contained in $N^{\prime}$ $-f(S)$ and meet each component of $N^{\prime}-f(S)$.

EXAMPLE. To see the necessity of the hypothesis that $f$ be proper, even in the situation where $S$ is compact, consider a wild $n$-cell $B$ in $E^{n}$ such that $\mathrm{Bd} B$ separates the two boundary components of the annulus $A$ given by

$$
A=\left\{\left(x_{1}, \ldots, x_{n}\right) \in E^{n} \mid 1 \leqq x_{1}^{2}+\cdots+x_{n}^{2} \leqq 4\right\} .
$$

Let $N^{\prime}=S^{n-1} \times S^{1}$ be obtained from $A$ by identifying the two boundary

2 Cernavskii [3, Appendix 2] has announced an affirmative solution in case $n \geqslant 5$. 
components of $A$ in the natural way. Now it is a simple matter to define a map $f$ of $E^{n}-B$ to $N^{\prime}$ such that, for the component $S$ of $\partial A$ contained in $E^{n}-B, f \mid S$ is $1-1, F(S)$ equals the image of $\mathrm{Bd} B$ in $N^{\prime}$, and $f\left(\left(E^{n}-B\right)-S\right)$ $=N^{\prime}-f(S)$.

For completeness we mention the following theorem. The key to the argument is that $N-S$ is $k$-LC at each point of $S$ if it is 1-LC [11, Theorem 6].

TheOREM 7. Suppose $S$ is a $(n-1)$-manifold in an n-manifold $N$. Then $N-S$ is 1-LC at each point of $S$ iff $S$ has a singular regular neighborhood. Furthermore, in case $N-S$ has two components, $U$ and $V, U$ is 1-LC at each point of $S$ iff $S$ has a singular regular neighborhood from $U$.

We close with two questions pertaining to an $(n-1)$-manifold $S$ in $E^{n}$ and a component $U$ of $E^{n}-S$. The second question is unsolved even for $n=3$, while the first has an affirmative solution in that case [15, Theorem 6].

Question 1. Suppose for each open subset $W$ of $S$ and each continuous function $\varepsilon: W \rightarrow(0, \infty)$ there exists a map $f: W \rightarrow U$ such that $\rho(w, f(w))$ $<\varepsilon(w)$ for each $w \in W$. Is $U$ l-LC at each point of $S$ ?

Question 2 (Free Surface Problem). If for each map $\varepsilon: S \rightarrow(0, \infty)$ there exists a map $f: S \rightarrow U$ such that $\rho(s, f(s))<\varepsilon(s)$ for all $s \in S$, is $C 1$-LC at each point of $S$ ?

\section{REFERENCES}

1. R. H. Bing, A surface is tame if its complement is 1-ULC, Trans. Amer. Math. Soc. 101 (1961), 294-305. MR 24 \# A1117.

2. J. W. Cannon, ULC properties in neighbourhoods of embedded surfaces and curves in $E^{3}$, Canad. J. Math. 25 (1973), 31-73. MR 47 \#2589.

3. A. V. Cernavskiī, The equivalence of local flatness and local 1-connectedness for imbeddings of $(n-1)$-dimensional manifolds in n-dimensional manifolds, Mat. Sb. 91 (133) (1973), 276-286 = Math. USSR Sbornik 20 (1973), 297-304.

4. R. J. Daverman, Locally nice codimension one manifolds are locally flat, Bull. Amer. Math. Soc. 79 (1973), 410-413. MR 47 \#9628.

5. S. Eilenberg and N. E. Steenrod, Foundations of algebraic topology, Princeton Univ. Press, Princeton, N. J., 1952. MR 14, 398.

6. D. B. A. Epstein, The degree of a map, Proc. London Math. Soc. (3) 16 (1966), 369-383. MR $33 \# 700$.

7. John Hempel, A surface in $S^{3}$ is tame if it can be deformed into each complementary domain, Trans. Amer. Math. Soc. 111 (1964), 273-287. MR 28 \#3409.

8. N. Hosay, The sum of a real cube and a crumpled cube is $S^{3}$, Notices Amer. Math. Soc. 10 (1963), 666; Errata, ibid. 11 (1964), 152. Abstract \#607-17.

9. W. Hurewicz and H. Wallman, Dimension theory, Princeton Math. Ser., vol. 4, Princeton Univ. Press, Princeton, N. J., 1941. MR 3, 312.

10. L. L. Lininger, Some results on crumpled cubes, Trans. Amer. Math. Soc. 118 (1965), 534-549. MR 31 \#2717.

11. M. H. A. Newman, Local connection in locally compact spaces, Proc. Amer. Math. Soc. 1 (1950), 44-53. MR 11, 453.

12. Paul Olum, Mappings of manifolds and the notion of degree, Ann. of Math. (2) 58 (1953), 458-480. MR 15, 338.

13. T. M. Price and C. L. Seebeck, III, Somewhere locally flat codimension one manifolds with 1-ULC complements are locally flat, Trans. Amer. Math. Soc. 193 (1974), 111-122.

14. E. H. Spanier, Algebraic topology, McGraw-Hill, New York, 1966. MR 35 \#1007.

15. Warren White, Some tameness conditions involving singular disks, Trans. Amer. Math. Soc. 143 (1969), 223-234. MR 40 \#2040. 
16. J. L. Bryant and R. C. Lacher, Embeddings with mapping cylinder neighborhoods, Topology 14 (1975), 191-201.

Department of Mathematics, University of Tennessee, Knoxville, Tennessee 37916 (Current address)

Department of Mathematics, University of Utah, Salt lake City, Utah 84112 